\title{
Video Article \\ Novel Object Recognition and Object Location Behavioral Testing in Mice on a Budget
}

\author{
Jiyeon K. Denninger ${ }^{1}$, Bryon M. Smith ${ }^{1}$, Elizabeth D. Kirby ${ }^{1,2,3}$ \\ ${ }^{1}$ Department of Psychology, Ohio State University \\ ${ }^{2}$ Department of Neuroscience, Ohio State University \\ ${ }^{3}$ Center for Chronic Brain Injury, Ohio State University
}

Correspondence to: Elizabeth D. Kirby at kirby.224@osu.edu

URL: https://www.jove.com/video/58593

DOI: doi:10.3791/58593

Keywords: Behavior, Issue 141, Memory, cognitive testing, object location, novel object recognition, mouse, hippocampus

Date Published: 11/20/2018

Citation: Denninger, J.K., Smith, B.M., Kirby, E.D. Novel Object Recognition and Object Location Behavioral Testing in Mice on a Budget. J. Vis. Exp. (141), e58593, doi:10.3791/58593 (2018).

\section{Abstract}

Ethologically relevant behavioral testing is a critical component of any study that uses mouse models to study the cognitive effects of various physiological or pathological changes. The object location task (OLT) and the novel object recognition task (NORT) are two effective behavioral tasks commonly used to reveal the function and relative health of specific brain regions involved in memory. While both of these tests exploit the inherent preference of mice for the novelty to reveal memory for previously encountered objects, the OLT primarily evaluates spatial learning, which relies heavily on hippocampal activity. The NORT, in contrast, evaluates non-spatial learning of object identity, which relies on multiple brain regions. Both tasks require an open-field-testing arena, objects with equivalent intrinsic value to mice, appropriate environmental cues, and video recording equipment and the software. Commercially available systems, while convenient, can be costly. This manuscript details a simple, cost-effective method for building the arenas and setting up the equipment necessary to perform the OLT and NORT. Furthermore, the manuscript describes an efficient testing protocol that incorporates both OLT and NORT and provides typical methods for data acquisition and analysis, as well as representative results. Successful completion of these tests can provide valuable insight into the memory function of various mouse model systems and appraise the underlying neural regions that support these functions.

\section{Video Link}

The video component of this article can be found at https://www.jove.com/video/58593/

\section{Introduction}

Effective cognitive tests isolate and assess the neural function of specific brain regions by examining behavior in a controlled environment ${ }^{1}$. In humans, specific tasks have been designed to assess the performance of targeted brain regions, such as the Wisconsin card sorting task for prefrontal function or the paired associates learning test of the Cambridge Neuropsychological Test Automated Battery (CANTAB) for hippocampal function ${ }^{2,3}$. These tests are designed to study the functions of specific brain regions in humans by assessing behaviors that result from the neural activity of those regions. The end goal of most biomedical research is the improvement of human health; however, many studies of brain function in health or disease cannot be ethically performed with human participants. For studies that cannot use human participants, small rodents such as mice are often the model of choice. Using mouse models allows for the direct control over experimental manipulations including alteration of gene expression, induction of injury or even modulation of circuit activity through optogenetic techniques. Behavioral testing of mice, similar to human testing, aims to assess the effect of experimental variables on brain function by measuring behaviors that rely on specific regions.

The hippocampus is an essential structure for memory formation in humans and rodents ${ }^{4}$. More specifically, the hippocampus plays a critical part in declarative memory involving relational representations, but not procedural memory, which relies on the motor centers of the brain ${ }^{4}$. Hippocampal memory function has been a focus of study across many fields of neuroscience because it is exquisitely sensitive to perturbation. Negative perturbations ranging from prolonged stress and aging to seizures and stroke are associated with hippocampal damage ${ }^{5}$. In contrast, positive interventions, such as social interaction, physical environmental enrichment, or exercise, improve hippocampal function ${ }^{6,7,8}$. Rodent studies with proper testing of hippocampal memory can reveal insight into the cellular and molecular mediators of memory as well as the effects of different environmental interventions on hippocampal function.

In rodents, several tests have been developed to study the hippocampus-dependent learning and memory ${ }^{9,10,11}$. They can be broadly subdivided into tasks that require a stimulus with emotional valence to elicit a change in behavior, and tasks that draw on the rodent preference to investigate novel stimuli ${ }^{11}$. Contextual fear conditioning, for example, pairs an unpleasant stimulus (foot shock) with an environmental context and then later tests memory for the context by measuring fear-induced freezing behavior ${ }^{9,11}$. The Morris water maze and its dry counterpart, the Barnes maze, use negative external reinforcement to promote spatial learning ${ }^{4,11}$. In each case, rodents seek escape from an aversive situation, being immersed in cold water or exposed on a brightly-lit platform, respectively. The radial arm maze, in contrast, relies on positive reinforcement as animals use natural foraging behavior coupled with spatial memory to retrieve small food rewards ${ }^{4,11}$. These tasks are widely used and have 
yielded foundational knowledge about hippocampal memory. However, negative and positive external reinforcements or fear-inducing stimuli like shock add an emotional component to these behavioral tests which in some cases may be undesirable. For example, the dorsal and ventral hippocampi are associated with distinct functions, spatial memory versus emotional regulation, respectively ${ }^{12}$. Tests that rely on an emotional response to stimuli may not accurately reflect impaired spatial memory if ventral hippocampal emotional regulation functions are also affected.

The OLT is a simple and effective test that provides a measure of hippocampus-dependent spatial memory ${ }^{13}$. The task relies on an animal's intrinsic preference for novelty without additional external reinforcement and can therefore typically avoid complications associated with differential emotional responses ${ }^{13}$. The present protocol for OLT is presented for mice, but it is also effective in rats if the dimensions of the equipment are appropriately scaled. The protocol consists of acclimating a mouse to an open-field-testing arena and then allowing it to investigate 2 objects in relation to spatial environmental cues. The mouse is then removed from the arena, and during a delay (inter-trial interval or ITI), one of the objects is moved. After the ITI, the mouse is reintroduced to the arena and allowed to freely explore. In general, mice prefer novelty, and if they remember the location of the objects from their initial exposure, they will spend more time investigating the moved object. Animals with hippocampal lesions have impaired spatial contextual learning and consequently demonstrate no preference for objects in the novel location $^{14,15}$.

The OLT can be used independently or in combination with an additional test of memory that draws on neural activity from multiple brain regions, the novel object recognition task (NORT). The NORT is identical to the OLT until the test phase, when one of the objects is replaced by a novel object instead of being moved to a new location. As is the case with the OLT, mice with good memory of the objects will spontaneously prefer investigating the novel object. In contrast to object location memory, which relies heavily on hippocampal substrates, object recognition memory appears to rely on a variety of brain regions and the involvement of the hippocampus is unsettled. Many studies report that hippocampal lesions or inactivation do not affect novel object preference ${ }^{10,13,16,17}$, while others find the opposite ${ }^{18,19}$. However, it is still a commonly used task to evaluate general memory function in rodents.

The protocol presented here delineates the steps involved in initiating and executing the OLT and NORT, both of which use an open-field-testing arena. Commercially available behavioral testing equipment can be cost-prohibitive, particularly for smaller labs. This protocol includes the design and simple steps to build arenas in-house at the minimal cost and without specialized tools. Furthermore, this protocol details the ideal behavioral testing area, including placement of arenas, contextual cues, and video recording system that sets the stage for implementation of the OLT and NORT protocols. Representative results for successful as well as flawed studies are presented, emphasizing the importance of optimizing all materials and procedures for each study.

\begin{tabular}{|l|l|l|}
\hline Description & Option & Quantity \\
\hline $\begin{array}{l}\text { Part A: Acrylic sheet - Opaque White }(0.635 \mathrm{~cm} \\
\text { x } 40 \mathrm{~cm} \text { x } 40.64 \mathrm{~cm})\end{array}$ & Routed Edges & 2 \\
\hline $\begin{array}{l}\text { Part B: Acrylic sheet - Opaque White }(0.635 \mathrm{~cm} \\
\text { x } 40 \mathrm{~cm} \text { x } 41.91 \mathrm{~cm})\end{array}$ & Routed Edges & 2 \\
\hline $\begin{array}{l}\text { Part C: Acrylic sheet - Opaque White }(0.635 \mathrm{~cm} \\
\text { x } 41.91 \mathrm{~cm} \text { x } 41.91 \mathrm{~cm})\end{array}$ & Routed Edges & 1 \\
\hline Acryllic Cement $(1 \mathrm{pt})$ & NA & 1 \\
\hline 16 Gauge Hypo Applicator & NA & 1 \\
\hline Combination Square & NA & 1 \\
\hline HD Webcam & NA & 1 \\
\hline Video Capture Software & NA & 1 \\
\hline USB 2.0 Extension Cable & NA & 1 \\
\hline Cable Conduit & NA & 1 \\
\hline
\end{tabular}

Table 1: Itemized list of materials and equipment required for behavioral testing.

\section{Protocol}

The following protocol has been approved by the Institutional Animal Care and Use Committee (IACUC) at the Ohio State University (OSU).

\section{Building the Arenas}

1. Order the materials outlined in Table 1: five sheets of acrylic, acrylic cement, and a $16 \mathrm{G}$ hypo applicator.

2. Wear appropriate safety equipment according to manufacturer's instructions, which may include eye, skin, and other types of protection.

3. Remove the protective paper coating from the acrylic sheets.

4. Dry fit all the materials to confirm that sizes are correct (Figure 1A).

5. Assemble and load the syringe with acrylic cement.

6. Align the long edge of an outside wall (Part B) with a top edge of the base (Part C) and ensure that they are perpendicular to each other using a combination or machinist square.

7. Using the syringe, apply a small and steady bead of cement directly to the corner of the two pieces being joined.

8. Hold the two pieces (Parts B and C) in place until they are initially set (approximately $5 \mathrm{~min}$ ). NOTE: Typically, they will be $80 \%$ hardened in $24 \mathrm{~h}$, but assembly can continue after $5 \mathrm{~min}$.

9. Repeat steps 1.5-1.7 with the other outside wall (Part B) to the same base 
10. Attach the two inner walls (Part A), one at a time, to the base using steps 1.5-1.7.

11. Additionally, use the syringe to apply a small and steady bead of cement directly to the corner now being formed by the outside wall (Part $B$ ) and the inside wall (Part A).

12. Hold these pieces in place for $5 \mathrm{~min}$.

13. After $24-48 \mathrm{~h}$, proceed to setting up the behavioral testing environment.

\section{Setting up the Behavioral Testing Environment and Equipment}

1. Place environmental cues (as described in the discussion section) across from each other and facing the arenas in the testing area (Figure 1B).

2. Arrange the four testing arenas in a 2-by-2 manner either on the floor or sturdy table at appropriate distances from the cues and the camera to maximize visual input to the mice (Figure 1B).

3. Verify the line of sight using a meter stick propped from each arena floor over the wall towards the cues to confirm that these distances between the arenas and the cues are appropriate.

4. Determine the optimal optical path length that allows video documentation of all four arenas by adjusting the height of the camera or the height of the table. (Figure 1B).

5. Connect the camera to a USB Extension Cable.

6. Using cable raceways, run the cable across the ceiling and down a wall to a computer running video capture software.

7. Hide the computer behind a curtain that will separate the mice in the testing area from the researcher (Figure 1C).

8. Assemble 4 each of at least 3 different objects that are approximately $2-5 \mathrm{~cm}$ in length and width and up to $10 \mathrm{~cm}$ in height to use for testing (Figure 1D).

A

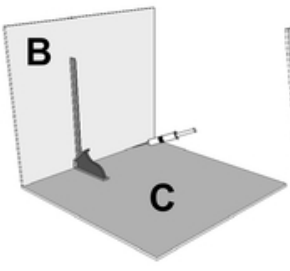

B

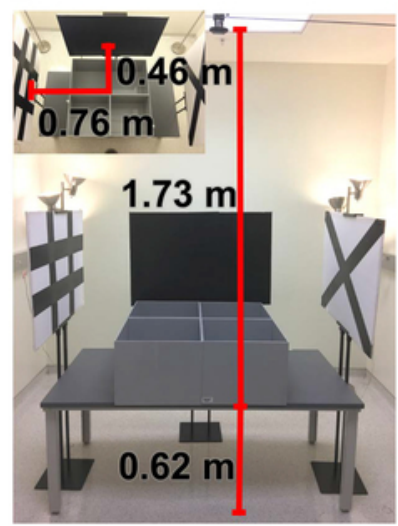

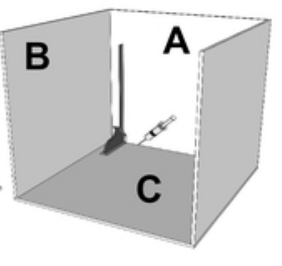

C

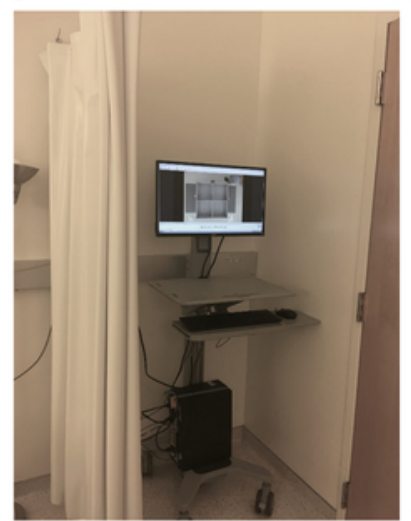

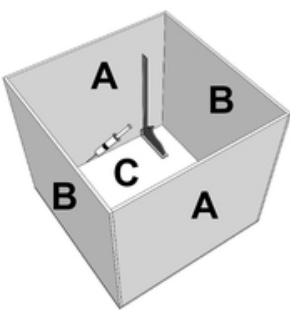

D

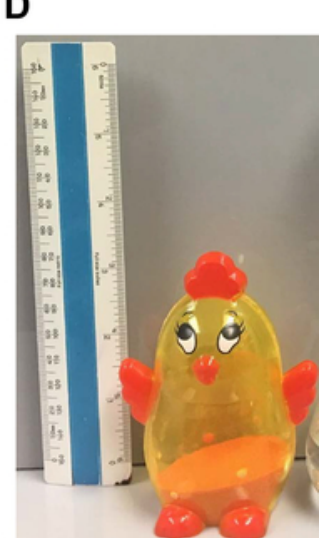

Figure 1: Behavioral testing preparation. (A) Open-field-testing arena assembly with part A corresponding to the inner wall, part B as the outer wall, and part $C$ as the base. The finished arena will have two outer walls (parts $B$ ) that run the entire edge of the base and two inner walls (parts A) that fit between the outer walls on the adjoining edges of the base (part C). All walls will rest on top of the base. (B) Representative arrangement of arenas on a $0.62 \mathrm{~m}$ high table, $60 \times 90 \mathrm{~cm}$ environmental cues, lights, and a camera for a testing area that allows the capture of all four arenas simultaneously. (C) A curtain hides the experimenter and computer system from mice during trials. The overhead lights are on for the purposes of taking this photograph, but during testing, only the floor lamps are on. Also, one of the environmental cues has been removed for this photograph of the testing area, but during testing, there is a fourth cue in front of the arenas, facing the all black cue behind the table. (D) A representative object (and ruler for scale) that is appropriate for OLT or NORT testing with mice. Please click here to view a larger version of this figure.

9. Validate these objects.

1. Obtain a minimum of 8 wild-type mice in the sex, strain, and age group representative of the experimental mice that will be used (e.g., 6 to 9 week-old female and male C57BI/6 mice).

2. Handle all mice daily for 1 min over the course of 3-5 days prior to testing.

3. Divide mice into groups of 4 and, if they are not already singly housed, move them into individual clean holding cages.

4. Bring them into the testing room and allow them to acclimate for at least $30 \mathrm{~min}$.

NOTE: Presence of the experimenter in the room for these $30 \mathrm{~min}$ will reduce stress on the mice during the task, particularly if the experimenter is male ${ }^{20}$.

5. After acclimation is done and the experimenter is ready to start, begin recording the video.

6. Place each mouse facing the walls of one corner of the arena (called the release corner) (Figure 2A). 
7. Allow the mice to explore the arenas freely for $10 \mathrm{~min}$.

8. Stop recording the video.

9. Return mice to their clean holding cages for a duration of $20 \mathrm{~min}$.

10. Clean all arenas with animal facility recommended cleaning methods, such as wiping with $70 \%$ ethanol to minimize olfactory cues before the next use.

11. Using double-sided tape, affix 2 different objects near 2 non-release corners such that the objects are counterbalanced in the arena, and $6 \times 6 \mathrm{~cm}^{2}$ from each wall of that corner (Figure 2B).

12. Start recording the video.

13. Place each mouse facing the walls in the release corner.

14. Allow mice to investigate the arena and objects freely for $10 \mathrm{~min}$.

15. Stop recording the video.

16. Place mice back in their clean holding cages for a duration of $20 \mathrm{~min}$.

17. Clean all arenas and objects with animal facility recommended cleaning methods such as wiping with $70 \%$ ethanol to minimize olfactory cues.

18. Repeat training trials with 2 new objects affixed in the same locations until all the objects (at least three different objects if conducting both the OLT and NORT) have been tested with each mouse.

19. Exclude objects that allow mice to sit on top of the object.

20. Analyze investigation time of each mouse with each object according to step 4 .

21. Exclude objects that have a negative or positive intrinsic value.

\section{Conducting the Behavioral Test}

1. One week prior to testing: familiarization to individuals conducting the behavioral tests

1. Handle adult 6 to 9 week-old female and male C57BI/6 mice daily for 1 min over the course of $3-5$ days prior to testing.

2. Day 1: habituation sessions

1. Divide mice into groups of 4 and, if they are not already singly housed, move them into individual clean holding cages.

2. Bring them in to the testing room and allow them to acclimate to the testing room for at least $30 \mathrm{~min}$.

NOTE: Presence of the experimenter in the room for these $30 \mathrm{~min}$ will reduce stress on the mice during the task, particularly if the experimenter is male ${ }^{20}$.

3. After acclimation is done and the experimenter is ready to start, begin recording the video.

4. Place each mouse in the arena (one mouse per arena) facing the walls of the release corner (Figure 2A).

5. Allow the mice to explore the arenas freely for $6 \mathrm{~min}$.

6. Stop recording the video.

7. Return mice to their clean holding cages during the inter-trial interval (ITI).

8. Clean all arenas with animal facility recommended cleaning methods such as wiping with $70 \%$ ethanol to minimize olfactory cues.

9. Repeat steps 3.3-3.9 two more times for a total of 3 habituation sessions for each mouse.

10. Return all mice to their home cages.

11. Clean all arenas with animal facility recommended cleaning methods such as wiping with $70 \%$ ethanol to minimize olfactory cues before use the next day.

12. Day 2: training trial, OLT, NORT

NOTE: The NORT is an optional test.

1. After $24 \mathrm{~h}$, bring the same group of mice in to the testing room and allow them to acclimate to the testing room for at least $30 \mathrm{~min}$ as done before the habituation sessions on the previous day.

2. Conduct a training trial using 2 objects placed in the arena (Figure 2B).

1. Using double-sided tape, affix objects $6 \times 6 \mathrm{~cm}^{2}$ away from 2 non-release corners such that they are counterbalanced in the arena.

2. Start recording the video.

3. Place each mouse facing the walls of the release corner as done during the habituation sessions.

4. Allow mice to investigate the arena and objects freely for $10 \mathrm{~min}$.

5. Stop recording the video.

6. Place mice back in their clean holding cages for an ITI of 20 min.

7. Clean all arenas and objects with animal facility recommended cleaning methods such as wiping with $70 \%$ ethanol to minimize olfactory cues.

3. Perform the OLT.

1. Move one of the objects used in the training trial to a new non-release corner and affix the object $6 \mathrm{~cm}$ from each wall of that corner with double-sided tape (Figure 2C).

NOTE: The other object should remain where it was during the training trial.

2. Start recording the video.

3. Place each mouse facing the walls in the release corner.

4. Allow mice to investigate the objects for $10 \mathrm{~min}$.

5. Stop recording the video.

6. Place mice back in their clean holding cages for an ITI of $20 \mathrm{~min}$.

7. Clean all arenas and objects with animal facility recommended cleaning methods such as wiping with $70 \%$ ethanol to minimize olfactory cues.

4. Perform the NORT. 
1. Replace the object that was not moved during the OLT with a novel object and affix the novel object $6 \mathrm{~cm}$ from the two walls of the corner with double-sided tape (Figure 2D).

2. Start recording the video.

3. Place each mouse facing the walls in the release corner.

4. Allow mice to investigate the objects for $10 \mathrm{~min}$.

5. Stop recording the video.

6. Place mice back in their home cages.

7. Clean all arenas and objects with animal facility recommended cleaning methods such as wiping with $70 \%$ ethanol to minimize olfactory cues before next use.

A

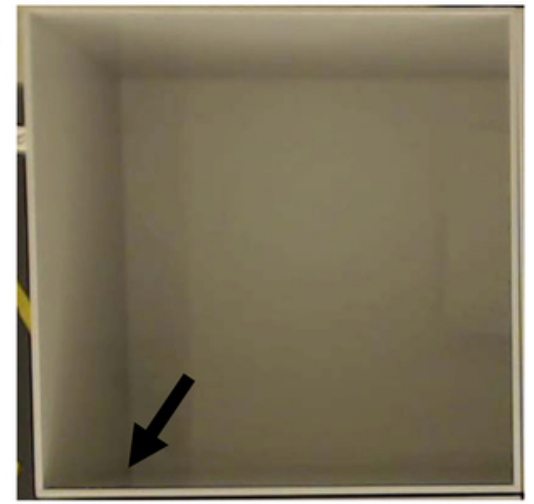

C

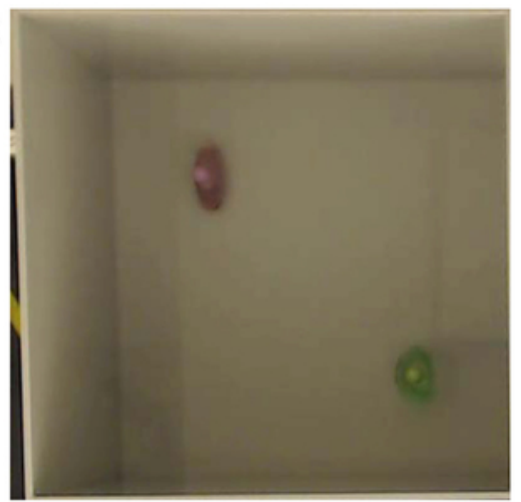

B

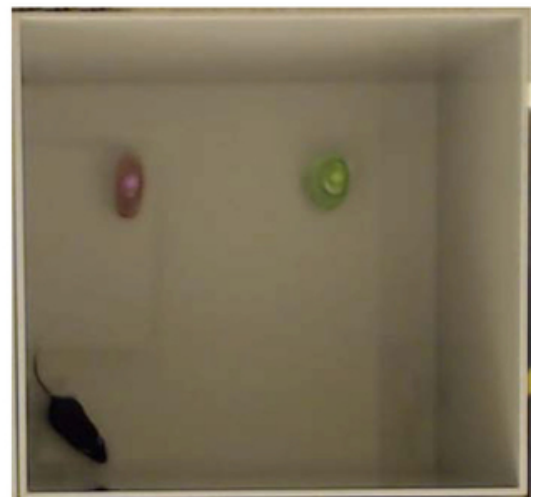

D

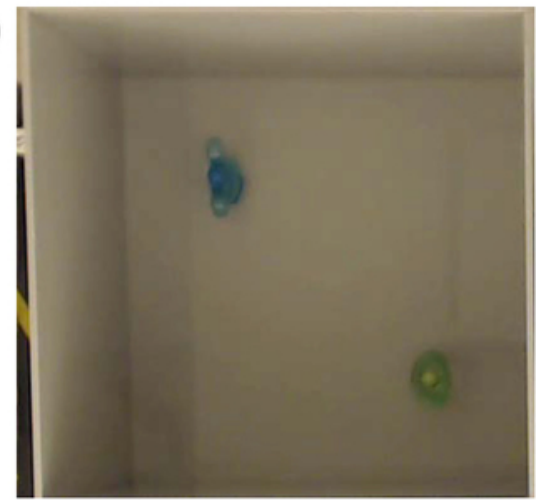

Figure 2: Arena configuration for trials. (A) Open-field testing arena without objects for habituation session. The black arrow indicates a release corner. This corner should be the same relative location in each arena and be consistent for every mouse being tested and for every trial. (B) For the training trial, two different objects are secured to the open field at $6 \times 6 \mathrm{~cm}$ away from their respective walls. (C) For the OLT, one object is moved to a new location, also $6 \times 6 \mathrm{~cm}$ away from the walls and not the release corner. (D) For the NORT, the object that was stationary in the OLT is replaced with a novel object while the moved object from the OLT is now the familiar object. Please click here to view a larger version of this figure.

\section{Analyzing Behavioral Test Data}

NOTE: Analysis of video should ideally be completed by at least two independent, blinded experimenters.

1. Open the video file.

2. Apply a transparent circle that provides a border of $2 \mathrm{~cm}$ around each object over the screen to help determine active investigation. Use a video file image with a ruler placed in an arena to calibrate this grid.

3. Observe mouse behavior and record the times the mouse is actively investigating the object, which consists of its nose pointed at the object at a maximum distance of $2 \mathrm{~cm}$ from that object.

1. Record the time stamp the mouse starts to investigate an object and the time stamp when it stops investigating that object.

2. Repeat this for both objects in the arena for the duration of the trial.

3. Calculate the cumulative time the mouse investigated each object by subtracting the start time from the stop time for each instance of object investigation, and adding all of those values.

4. Calculate percent of total investigation time or the discrimination index with the following formulas:

1. Calculate percent of total investigation time $=$

$\frac{\text { (time with novel location or object) }}{\text { (time with novel location or object }+ \text { time with familiar location or object) }} \times 100$ 
NOTE: A value above $50 \%$ indicates greater investigation of the novel location or object.

2. Calculate discrimination index $=$

time with novel location or object - time with familiar location or object

time with novel location or object + time with familiar location or object

NOTE: A positive value indicates more time investigating the novel object. A discrimination index of zero indicates equal time spent with both objects.

5. Graphically represent results and complete statistical analyses using a t-test or ANOVA as appropriate for the number of groups being compared.

\section{Representative Results}

Figure 3 provides examples of typical positive and negative results obtained with male and female adult C57BI/6 mice using this protocol ${ }^{6}$. Interpretation of OLT and NORT data always applies to the aggregate data of a group (see discussion below). Investigation time for a single mouse cannot be interpreted as memory or lack of memory. However, the performance of a group of mice (i.e., multiple samples) can be compared to other groups or to the fixed chance levels using statistical testing. During a typical training trial, groups of mice do not show a significant preference on average for either of the objects as they are both equally novel and do not have any intrinsically negative or positive value to the mice (Figure 3A and 3B). If the aggregate data of a group of mice show significant preference for one object over another during training, these objects should not be used because that inherent preference/aversion will confound results in the subsequent trials. Additionally, the total investigation time of all the mice must meet a minimum standard (traditionally set at 20 seconds $^{21}$ ) and should be compared to ensure that there is no baseline difference in investigation that may affect subsequent tests of memory.

During the OLT, memory for object location is reflected by mice spending on average significantly more than $50 \%$ of total investigation time with the moved object (Figure 3A). If the total investigation times of the individual mice vary greatly, results are better depicted as a discrimination index for the objects (Figure 3B). The significant increase in average discrimination index in Figure 3B indicates that the mice spent more time with the object after it had moved. Whether measured by increase in percent time or discrimination index, the increase in investigation of the object after it is moved suggests that the mice remember where the object was located during training.

The last trial of this protocol assesses object recognition memory. A representative example with one group of mice demonstrates a higher average percentage of investigation time (Figure 3C) as well as positive discrimination index (Figure 3D) compared to the fixed control values of $50 \%$ and 0 , respectively. As with the OLT data, if there is significant variability in total investigation time between individual mice, the discrimination index is likely the better method to visualize this data. Figure 3E shows an example of a 2-group comparison in the NORT and some of the statistical complications that can arise in these tests (see discussion). While a one-sample t-test for group $B$ shows investigation significantly above $50 \%$, the same test for group $A$ does not. This finding does not mean that $A$ and $B$ are different from each other. To determine group differences, a separate two-sample non-parametric Mann-Whitney test comparing groups B to A must be performed. A two-sample nonparametric Mann-Whitney test of this representative group data shows no significant difference $(p=0.66)$ between the two groups in percentage of novel object investigation time.

Both the OLT and NORT are highly sensitive to the intrinsic value of objects and thorough testing of object equivalence is necessary to ensure that there is no intrinsic bias that can confound results. Figure $\mathbf{3 F}$ and $\mathbf{3 G}$ show an example of inappropriate object selection. In a pilot test with a sample size of 4 , mice showed a trend towards spending less than $50 \%$ of investigation time with object A when paired against object $B$ (Figure 3F). When these objects were then used in a NORT with object $A$ as the novel object and a larger sample size of 16 , mice spent significantly less than $50 \%$ of investigation time with object $A$ (Figure $\mathbf{3 G}$ ). This aversion to the novel object is easily recognizable here as a technical flaw in the experiment and exemplifies why pilot testing of objects for inherent preference/aversion is essential. 

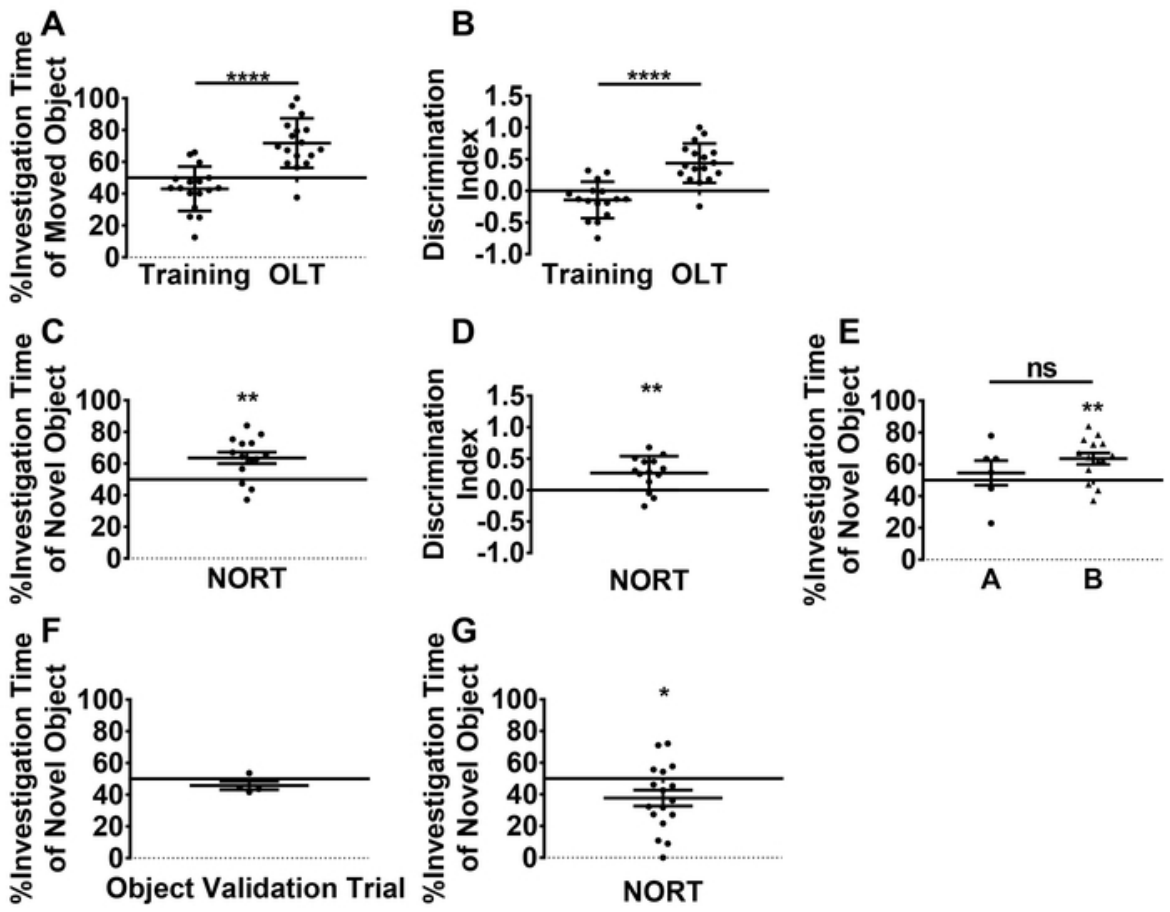

Figure 3: Behavioral testing data with wild type adult C57BI/6 mice. (A) Comparison of percent total investigation time of the moved object during the training trial versus the OLT shows significant increase in investigation, after the object moved. ${ }^{* * * *} p<0.0001$, paired t-test. (B) Representative results for moved object investigation during training and OLT trials displayed as a discrimination index similarly show a significant increase in investigation of the object after it is moved. ${ }^{* * *} \mathrm{p}<0.0001$, paired t-test. (C) Percent total investigation time of the novel object in the NORT shows significant preference for investigating the novel object. ${ }^{* *} p=0.0024$, one-sample t-test vs. $50 \%$. (D) Representative results for novel object investigation in the NORT displayed as a discrimination index similarly show preference for investigating the novel object. ${ }^{* *} p=0.0024$, one-sample t-test vs 0 . (E) Representative results of a NORT analysis involving two different groups of mice. Group B differs significantly from $50 \%$ by one-sample t-test ( $\left.{ }^{* *} p=0.0024\right)$, but group A does not $(p=0.5837)$. In a separate analysis, to compare groups, a twosample Mann-Whitney test is used because of the uneven group sizes and no significant difference in investigation is found ( $p=0.66$, ns). $(F)$ Percent time with an object during validation trials in a small sample size shows a trend towards aversion to the object. $p=0.2159$, one-sample t-test. (G) With a larger sample size and the object from (F) used as a novel object in a NORT, a significant aversion to the object is found, even though it is the novel object. ${ }^{*} p=0.0270$, one-sample t-test. This is an example of a technical failure in object selection. Data are presented as mean \pm SEM. Data from panels $C$-E are adapted from a previous publication ${ }^{6}$. Please click here to view a larger version of this figure.

\section{Discussion}

This protocol provides a cost-effective method to conduct object location and novel object recognition behavioral testing in mice. These tests enable the evaluation of hippocampal function as well as function of other cortical regions, such as the prefrontal cortex, involved in object recognition ${ }^{10}$. The OLT and NORT have the advantage of avoiding stimuli with strong emotional valence that are required for the Morris water maze, contextual fear conditioning, Barnes maze or radial arm maze. They also avoid the need for food deprivation as required for the radial arm maze. Furthermore, this protocol describes a simple two-day testing procedure that does not require extensive or complicated equipment for execution or analysis. One disadvantage of these tasks is that they do not allow for measures of learning or acquisition. A difference in novelty investigation could be due to poorer learning about objects during training, poorer memory for what was learned or both. Total time spent investigating objects is an important measure for ruling out any inherent differences in exploration drive but is not a measure of learning. If measures of learning are important for an experimental question, a water maze, Barnes maze, or radial arm maze would likely be preferable.

Custom building of behavioral arenas has the potential to save hundreds of dollars and bring object testing within the financial reach of a wide variety of labs. This protocol eliminates many obstacles and streamlines the process of fabrication to make in-house arena construction more accessible to scientists with no specialized training in acrylics. It is important to note that purchasing colored acrylic sheets that will contrast with the mice, such as white acrylic for black mice and black acrylic for white mice, will facilitate data acquisition and analysis, especially when using commercially available analysis software. Ordering cut-to-size sheets with "routed edges" eliminates the need for a table saw (Table 1), and the use of acrylic cement removes the need to drill and countersink pilot holes. Screwing in fasteners, drilling, and cutting acrylic often causes it break, chip and crack due to its brittle nature. Because the cement is a solvent, it will flow into the area being joined, dissolving and softening any acrylic it encounters. Thus, it should not be applied to each piece separately as if it were a traditional glue. Unlike glue, the cement will not fill negative spaces or adhere to surfaces. This is the primary reason to order "routed edges" as this will ensure a smooth and flat edge, creating a much better bond. When the cement dries, it will have fused the two acrylic sheets into a single piece in a process called "solvent welding". Much like metal welding, the finished product is a single piece, but the welded area will always remain the weakest location. As such, once the arenas are in use, care should be taken to avoid direct impact or extreme stress at those junctures. 
This protocol also shows how to set up 4 arenas for simultaneous testing of up to 4 mice (Figure 1 and Figure 2). The opaque walls of the arenas prevent mice from seeing one another during testing, but there is still a possibility that having other mice in the room causes odor or noise distractions that can impair testing. Habituation trials as detailed here can help mitigate this concern, as mice are exposed to the multi-animal room conditions before behavioral testing. However, if distractions from other mice or experimenter noise associated with handling other mice is a strong concern, one arena with one mouse can be used, as well, though it will increase the time required to complete the OLT and NORT with multiple mice. More than 4 arenas could theoretically be used as well, but most cameras do not have a wide enough field of view to show that many arenas with good resolution.

The dimensions and distances provided here are general guidelines for mouse behavioral testing in a typical testing room that is $16 \times 16 \mathrm{x} 16$ $\mathrm{m}^{3}$ in dimensions (Figure 1B and 1C). Set up of appropriate environmental cues, arenas, and video recording equipment must be optimized for each environment. Cues can consist of large shapes or patterns (typically in black and white) that enable mice to spatially orient themselves during the OLT. Instead of placing cues at different locations across from each other, cues can also be mounted to the walls of the testing area. This protocol recommends dividing the testing room with a curtain to hide the researcher and computer during behavioral testing. During all habituation, inter-trial intervals, and active trials, researchers should close the curtain to separate themselves from the testing area. If this is not practical, the computer can remain in view of the mice, but the researcher must move out of view during the task. If the researcher is present, the mice may try to rely on her or him as a spatial cue.

All behavioral testing should be completed in a temperature- and humidity-controlled environment with dim, but even illumination at around 310 lux and minimal extraneous sound or strong environmental odor cues, such as perfumes on experimenters. Between each trial and testing day, all arenas and objects should be cleaned with animal facility recommended methods of sterilization such as wiping with $70 \%$ ethanol or unscented bleach wipes to minimize olfactory cues. If chemical disinfectants are used, a final rinse with $70 \%$ ethanol is recommended because many chemical disinfectants can be irritating to the animals' feet. As with any behavioral task, handling mice for several days before testing is necessary to familiarize them with the individuals who will be performing the OLT and NORT and reduce stress during testing ${ }^{20,22}$. As mice can experience acute stress due to unfamiliar individuals in the vicinity of the testing area, it is also recommended that all behavioral testing should be completed by the same individual(s). The testing parameters and conditions detailed in this protocol have been optimized for 6 to 9 week-old adult C57BI/6 mice and would be most useful in revealing memory impairments due to injury in this age group or memory impairments due age itself in older mice. If the aim is to test for memory improvements in young mice, a longer ITI ranging from 1 hour to 1 day would be more appropriate to avoid ceiling effects on performance in the easier $20 \mathrm{~min}$ ITI version. Indeed, ITIs can range from 5 min (for immediate recall) to several hours or days (for remote memories), depending on the specific needs of the experiment and the strains and ages of the mice. Importantly, regardless of the length, all ITls should be consistent between sessions in an experiment. As different strains and ages of mice exhibit differences in behavior and learning, the timing for each trial and interval, testing area arrangement, and objects used can be modified according to the particular strain of mice, their age, and the specific injury/disease/intervention model being tested ${ }^{9,21,23,25}$.

While the hippocampal dependence of the spatial memory functions tested in the OLT are well-established, the NORT may or may not rely on the hippocampus. Interpretation of data from the NORT should take this caveat in to account. The determining variables, for whether the hippocampus is involved, in object recognition memory are not agreed upon yet, but could include ITI length or saliency of spatial cues ${ }^{18}$. Notably, the presented protocol uses spatial OLT before the NORT, which may bias mice towards using hippocampal processes in the NORT. Thus, it is important to note that the order can be reversed, or each task can be run independently, depending on the experimenters' questions and needs.

Object selection is a critical aspect of both the OLT and NORT ${ }^{22,24}$. Ideal objects are heavy enough not to be easily displaced by a mouse and made of material, like glass or metal, that a mouse cannot damage by chewing or scratching. Wooden, foam, or soft plastic objects are not appropriate as they are easily deformed and are difficult to keep odor-free. Furthermore, the objects used in the trials should all be relatively similar in size, texture, odor and material. Figure 1D gives an example of an appropriate object that could be used. This orange plastic figurine of a chick is filled with sand to give it enough weight and sealed to prevent leakage of cleaning reagents or other odor-causing agents. Because of the shape of the top, mice are unable to climb on top of or sit on this object. For the OLT or NORT, this object is best paired with another object of similar size, weight, material, color and complexity, such as a similar plastic figurine of a rabbit. To ensure that object investigation truly reflects preference for novelty, all objects must be validated for equivalent intrinsic value with a minimum of 8 mice in the same strain, sex, and age of the experimental group as described in section 2.9 of the protocol. Additionally, the objects should be randomized in terms of which object is the novel or moved object between mice in the same study to further ensure that inherent characteristics of the objects are not affecting preference. Object placement can also greatly affect the success or failure of the OLT and NORT. Objects must be counterbalanced in the arenas and not be too close to the walls. A corner crowded by an object is an attractive place for mice to hide and this will confound measures of investigation.

An important prerequisite to data collection is defining "active investigation", which is when a mouse engages an object with its nose pointed at the object no more than $2 \mathrm{~cm}$ away. A mouse moving over the top of the object or looking past the object does not qualify as active investigation. Furthermore, since the OLT and NORT depend on a mouse remembering either the spatial location or actual features of the object, there must be sufficient study of these during the training trials. Thus, the researcher must define a minimum investigation time and exclude any subjects that do not meet that baseline level of investigation, traditionally set at 20 seconds ${ }^{21}$.

Quantification of object investigation time can be accomplished in various ways either with or without expensive analysis software. If manual scoring is used, as described here, the most comprehensive data collection can be achieved by recording the time stamps on the video when the mouse is investigating the object. Recording the start and stop times of object investigation during the three individual trials creates a permanent log of unbiased time stamps and facilitates accurate manual calculation of data as opposed to alternative methods such as using a stop watch to additively record the total time a mouse investigates each object.

Commercial software packages are also available for scoring object investigation. Commercially available software can provide a wealth of data beyond the hand scoring data described here, including total distance traveled, amount of time spent in certain areas of the arena, and speed of movement ${ }^{16,23}$. Software, once properly calibrated and confirmed to reliably detect object investigation, can also yield data much faster than manual scoring. This faster data yield may, in the long run, yield net savings compared to the human-hours required for manual scoring. However, most software packages for behavioral analysis have high up-front costs that can be prohibitive for many labs and manual scoring can often be accomplished by student researchers, making the hourly costs of behavioral scoring minimal. Commercial software also frequently 
comes with restrictions on how many users can access the software concurrently, limiting the data throughput and time savings. Though there are many advantages to these software packages, they are not necessary to gather the essential information of time investigating objects from the OLT and NORT. The ability to manually acquire this data makes novel object tasks more financially accessible to a wider variety of researchers.

An additional feature of this protocol is that the first session of habituation is essentially a trial in an open field, which can yield data on activity and anxiety levels. Activity levels can be quantified using total distance traveled or average speed, if tracking software is available. Similarly, with tracking software, anxiety measures can be derived from time spent in the center of the arena or distance traveled in the center. These data can also be acquired manually by overlaying the arena video with a grid and quantifying crossings. This open-field data can help rule out gross motor deficits or excessive anxiety that might interfere with object investigation later.

The statistical tests used in this protocol are representative of traditional methods of analysis. When comparing 2 groups of mice, a two-sample, two-tailed t-test is recommended to test the significance of the difference in percent time investigating the moved or novel object between groups. If the sample sizes are uneven, have exceptionally high variability, or show uneven variability between groups, a non-parametric test is recommended instead, as many of the underlying assumptions of the t-test will be violated in these conditions. When comparing 3 or more groups of mice, ANOVAs are recommended to test whether group has a significant effect on percent time investigating the moved or novel object. Error-corrected post-hoc tests, such as Tukey's test or Bonferroni-corrected pairwise comparisons, can then be applied to test the difference in percent investigation time between each pair of groups. With the OLT, an additional way to analyze data is to test for a significant change in percent time investigating the moved object from training to OLT trials. With only one group of mice, this test would take the form of a paired, two-tailed t-test, testing for significant changes in percent time with the moved object from training to OLT in each mouse (keeping in mind that these are paired analyses and not independent measurements, since each mouse yields two data points). With two groups of mice, a repeated measures two-way ANOVA would be used, with the repeated measure being the trial (training versus OLT) and group assignment being the second factor. Post-hoc tests for differences between treatment groups should compare the groups to each other within trials.

Alternatively, if evidence of memory in the OLT or NORT is all that is being tested, a one-sample t-test of percent time investigating an object versus the fixed value of $50 \%$ can be used. A percent time significantly higher than $50 \%$ suggests memory for the object. A percent time significantly below $50 \%$ suggests aversion (for some reason), and object choices should be re-evaluated. However, the one-sample t-test cannot reveal whether two or more groups differ from each other. For example, in an experiment with two groups of mice, if Group A spent $60 \%$ of the time with the novel object for $p=0.049$ by one-sample t-test comparison to $50 \%$, and Group B only spent $59 \%$ of the time with the novel object for $p=0.051$, Group A is significantly different from $50 \%$ (and group B is not). However, it is erroneous to conclude that these two groups are significantly different from each other. A two-sample t-test comparing $A$ and $B$ can easily reveal that these two groups are statistically indistinguishable. If the end goal is to compare the memory performance of two or more groups, those groups must be statistically compared to each other, not only compared to an external standard. A similar guideline applies for a comparison of time spent with a moved object between training and OLT. In this case, finding a significant difference in percent time with a moved object between training and OLT in one group and not in another does NOT show that these groups are statistically different. Groups must be post-hoc compared against each other within each trial, not just across trials within group.

It is important to keep in mind that the investigation time of any individual mouse cannot be used as evidence for or against memory. Rather, memory for object location or identity in these tasks can only be concluded based on aggregate data that is statistically compared to either another group's aggregate data or the fixed chance levels ( $50 \%$ for time, 0 for discrimination ratio). The sample size needed will depend heavily on the effect size of a particular manipulation and variability in behavior, both of which in turn will depend on the mice being used. Age, sex, and manipulations all impact variability. In the example data presented in Figure 3A and 3B, $n=14$ subjects were used, yielding an effect size of 0.68 and power of 0.65 for a paired t-test with $\alpha=0.05$. If a power of 0.8 were desired for this comparison, a sample size of 18 would be required.

This discussion is framed around p-values and significance cutoffs because these are the measures and analyses most typically reported for OLT and NORT data, and therefore are likely to familiar to both experimenters and reviewers. This reliance on p-values has been heavily criticized as statistically invalid ${ }^{26}$. However, though alternative analysis methods exist and are endorsed in some journals ${ }^{27}$, none have been broadly adopted by the behavioral and biomedical fields as standard ${ }^{26}$.

In summary, this protocol effectively tests memory in mice at minimal costs. Recommendations for appropriate modifications to the protocol are included to ensure successful implementation with any small rodent model. Application of this protocol to specific injury or therapeutic intervention models can reveal valuable functional relevance that complements the cellular and molecular mechanisms being studied.

\section{Disclosures}

The authors have nothing to disclose.

\section{Acknowledgements}

This work was funded by R00 NS089938 from NIH and seed funding from Chronic Brain Injury and Discovery Themes at The Ohio State University to EDK.

\section{References}

1. Krakauer, J.W., Ghazanfar, A.A., Gomez-Marin, A., Maclver, M.A., Poeppel, D. Neuroscience Needs Behavior: Correcting a Reductionist Bias. Neuron. 93 (3), 480-490 (2017). 
2. Lange, F., Seer, C., Kopp, B. Cognitive flexibility in neurological disorders: Cognitive components and event-related potentials. Neuroscience and Biobehavioral Reviews. 83, 496-507 (2017).

3. Barnett, J.H., Blackwell, A.D., Sahakian, B.J., Robbins, T.W. The Paired Associates Learning (PAL) Test: 30 Years of CANTAB Translational Neuroscience from Laboratory to Bedside in Dementia Research. Current Topics in Behavioral Neuroscience. 28, 449-474 (2016).

4. Eichenbaum, H., Otto, T., Cohen, N.J. The hippocampus-what does it do? Behavioral and Neural Biology. 57 (1), 2-36 (1992).

5. Bartsch, T., Wulff, P. The hippocampus in aging and disease: From plasticity to vulnerability. Neuroscience. 19 (309), 1-16 (2015).

6. Smith, B.M., Yao, X., Chen, K.S., Kirby, E.D. A Larger Social Network Enhances Novel Object Location Memory and Reduces Hippocampal Microgliosis in Aged Mice. Frontiers in Aging Neuroscience. 10 (142), 1-16 (2018).

7. Chieffi, S., et al. Exercise Influence on Hippocampal Function: Possible Involvement of Orexin-A. Frontiers in Physiology. 14 (8), 85 (2017).

8. Garth, A., Roeder, I., Kempermann, G. Mice in an enriched environment learn more flexibly because of adult hippocampal neurogenesis. Hippocampus. 26 (2), 261-271 (2016).

9. Brown, R.E., Stanford, L., Schellinck, H.M. Developing standardized behavioral tests for knockout and mutant mice. Institute for Laboratory Animal Research Journal. 41 (3), 163-174 (2000).

10. Barker, G.R., Warburton, E.C. When is the hippocampus involved in recognition memory? Journal of Neuroscience. 31 (29), 10721-31 (2011).

11. Savage, S., Ma, D. Animal behavior testing: memory. British Journal of Anaesthesia. 113 (1), 6-9 (2015).

12. Fanselow, M.S., Dong, H.W. Are the dorsal and ventral hippocampus functionally distinct structures? Neuron. 65 (1), 7-19 (2010).

13. Vogel-Ciernia, A., Wood, M.A. Examining object location and object recognition memory in mice. Current Protocols in Neuroscience. 69:8 (31), 1-17 (2014)

14. Ammassari-Teule, M., Passino, E. The dorsal hippocampus is selectively involved in the processing of spatial information even in mice with a genetic hippocampal dysfunction. Psychobiology. 25 (2), 118-125 (1997).

15. Le Merrer, J., Rezai, X., Scherrer, G., Becker, J.A., Kieffer, B.L. Impaired hippocampus-dependent and facilitated striatum-dependent behaviors in mice lacking the delta opioid receptor. Neuropsychopharmacology. 38 (6), 1050-9 (2013).

16. Hattiangady, B., et al. Object location and object recognition memory impairments, motivation deficits and depression in a model of Gulf War illness. Frontiers in Behavioral Neuroscience. 8, 78 (2014).

17. Oliveira, A.M., Hawk, J.D., Abel, T., Havekes, R. Post-training reversible inactivation of the hippocampus enhances novel object recognition memory. Learning and Memory. 17 (3), 155-60 (2010).

18. Cohen, S.J., Stackman, R.W. Jr. Assessing rodent hippocampal involvement in the novel object recognition task. Behavioral Brain Research. 285, 105-117 (2014).

19. Cohen, S.J., et al. The Rodent Hippocampus Is Essential for Nonspatial Object Memory. Current Biology. 23 (17), 1685-1690 (2013).

20. Sorge, R.E., et al. Olfactory exposure to males, including men, causes stress and related analgesia in rodents. Nature Methods. 11 (6), 629-32 (2014).

21. Ennaceur, A., Delacour, J. A new one-trial test for neurobiological studies of memory in rats. 1: Behavioral data. Behavioral Brain Research. 31 (1), 47-49 (1988).

22. Leger, M., et al. Object recognition test in mice. Nature Protocols. 8 (12), 2531-7 (2013).

23. Wolf, A., Bauer, B., Abner, E.L., Ashkenazy-Frolinger, T., Hartz, A.M. A Comprehensive Behavioral Test Battery to Assess Learning and Memory in 129S6/Tg2576 Mice. Public Library of Science ONE. 11 (1), e0147733 (2016).

24. Ennaceur, A. One-trial object recognition in rats and mice: methodological and theoretical issues. Behavioural Brain Research. 215, 244-254 (2010).

25. Lueptow, L.M. Novel Object Recognition Test for the Investigation of Learning and Memory in Mice. Journal of Visualized Experiments. 126, e55718 (2017).

26. Wasserstein, R.L., Lazar, N.A. The ASA's Statement on p-Values: Context, Process, and Purpose. The American Statistician. 70 (2), $129-133$ (2016).

27. Ranstam, J. Why the P-value culture is bad and confidence intervals a better alternative. Osteoarthritis and Cartilage. 20 (8), $805-808$ (2012). 